\title{
RAMAN AND PHOTOLUMINESCENCE STUDIES ON THERMALLY ANNEALED POROUS SILICON
}

\author{
Auushree Roy ${ }^{+}$, K. Jayararn and A. K. Sood" \\ Department of Physics, Indian Institute of Science, \\ Bangalore 560 012, India. \\ + Also at Solid State and structural Chemistry Unit, \\ Indiac Institute of Science, Bangalore 560 012, India. \\ "Also at Jawaharlal Nehru Centre for Advanced Scientific Research. \\ IISc Campus, Bangalore 560 012, India.
}

\begin{abstract}
We report Raman and Photoluminescence (PI,) st udics of porous silicon (PS) as a. function of isochronal thermal annealing from room temperature to $900^{\circ} \mathrm{C}$. The $\mathrm{PL}$ peak position and intensity show nonmonotonic variation with increasing temperature. The PL intensity first increases upto $100^{\circ} \mathrm{C}$, then decreases till $550^{\circ} \mathrm{C}$ and recovers its intensity at $700^{\circ} \mathrm{C}$ before it completely disappears at $800^{\circ} \mathrm{C}$. The: red shifted asymmetric Raman line shape can be fitted by phonon confinement model along with the disordered silicon component. Our results clearly indicate that the origin of visible PL can be better explained by a new hybrid model which incorporates both nanostructures for quantum confinement and silicon complexes (such as $\mathrm{SiH}_{x}$ and siloxerie) and defects at $\mathrm{Si} / \mathrm{SiO}_{2}$ inlerlaces as luminescont centres.
\end{abstract}

\section{i. Introduction}

The recent observations of highly efficient visible photo- and electro- luminescence at room temperature from electrochemically etched porous silicon (PS) have stimulated a lot of excitement mainly due to the possibility of its use in Si-based optoelectronic applications. But till date, our understanding of the origin of the photoluminescence (PL) in PS is still incomplete. In one model, the quantum confinement effect on the silicon band structure arising from the formation of nanoscale units $(<50 \AA)$ in PS is responsible for the visible PL. The alternative model proposes that the PL is extrinsic to silicon and originats from silicon complexes like siloxene [1], hydride species $\mathrm{SiH}_{x}$ [2], polysilanes [3], or hydrogenated amorphous silicon [4].

The quantum confinement model, first suggested by Canham [5], is supported by transmission electron microscopy (TEM) studies and first principle electronic structure calculations for $\mathrm{H}$-terminated $\mathrm{Si}$ - wires (width 12 to $35 \AA$ ) [6]. The latter have shown that the band gap of the silicon wire increases and become direct at the zone centre. EPR measurements also supported the confinement model [7]. However, there are many experimental observations involving variations of the PL characteristics as a function of temperature, pressure, laser irradiation and post-preparation treatments which can not be explained in terms of the quantum confinement model. These are briefly mentioned as follows: (a) The temperature dependence of the PL band in PS is anomalous $[8,9]$. The peak position shows red as well as blue shift with increasing temperature. Rapid thermal oxidation experiments [10] have shown red shift of the peak till $650^{\circ} \mathrm{C}$ and then blue shift for $650^{\circ} \mathrm{C}<\mathrm{T}<$ $900^{\circ} \mathrm{C}$. The PL integrated intensity decreases between $200^{\circ} \mathrm{C}$ and $300^{\circ} \mathrm{C}$, and then recovers at $750^{\circ} \mathrm{C}$. (b) Prokes et al [11] have carried out experiments on thermal annealing of PS at $430^{\circ} \mathrm{C}$ followed by dipping it in HF acid. During the heating cycle, the PL intensity drops significantly and the peak shifts to $1.68 \mathrm{eV}$. The HF dipping restores the intensity and the peak value shifts to $1.90 \mathrm{eV}$. (c) Various groups have reported the contradicting results on the effect of the hydrostatic pressure on the peak position of the PL. Sood et al [12] have reported red shift of the peak position with increasing pressure whereas Zhou et al [13] observe a blue shift for low pressure and then a small red shift above $20 \mathrm{kbar}$. (d) Laser irradition causes a blue or a red shift of the PL peak position depending on the parameters of the sample preparation $[13,14]$. (e) Increasing porosity did not always correspond to a blue shift of the PL band [15]. (f) The expected correlation between red shift of the Raman line due to phonon confinement and the corresponding blue shift of the PL band in nanostructure is shown to be absent [12]. (g) Photoemission studies have shown presence of $\mathrm{Si}, \mathrm{O}$ and $\mathrm{F}$ in ion-etched PS [16].

Keeping in mind the incomplete, rather confused, understanding of the origin of the PL in PS, we have carried out simultaneous Raman and PL studies on PS as a function of isochronal thermal annealing from room 
temperature to $900^{\circ} \mathrm{C}$. Our other motivation to carry out thermal annealing studies came from the recent report of electroluminescence in diffused PS p-n junction, where the diffusion of phosphorous is carried out at $850^{\circ} \mathrm{C}$ for 30 minutes [17]. The earlier Raman measurements on PS [12] have shown that the phonon is red shifted with respect to the bulk silicon and the line shape is highly asymmetric on the low frequency scale. This has been attributed to confinement of the phonon in nanoscale silicon particles formed in the PS. Our objective of Raman measurement is to see the effect of thermal annealing on the nanoscale structures together with changes in luminescence characteristics. Our results support a new hybrid model [14] which incorporates features of both the models of quantum confinement as well as the silicon based compounds like $\mathrm{SiH}_{x}$ and $\mathrm{SiO}_{2}$ formation in the PS.

\section{Experimental}

The porous silicon was prepared by anodic etching of boron doped p-type Si wafers of $<100>$ orientation (resistivity $1 \Omega$-cm) in $48 \% \mathrm{HF}$ : ethanol (1:1) solution, keeping the current density between 10 and $15 \mathrm{~mA} / \mathrm{cm}^{2}$. $\mathrm{PL}$ and Raman spectra were recorded at room temperature in $45^{\circ}$ reflection geometry using $5145 \AA$ line of argon ion laser as excitation source and computer controlled SPEX Ramalog (model 14018) equipped with a cooled photomultiplier tube and a photon counting system. For thermal treatment in air, the sample was introduced in the temperature controlled furnace maintained at a desired annealing temperature $(\mathrm{T}$, ) and kept for 5 minutes. Then it was taken out of the furnace and cooled to room temperature to record both Raman and PL spectra at the same spot of the sample. The sample was annealed at seventeen different temperatures ranging from $25^{\circ} \mathrm{C}$ to $900^{\circ} \mathrm{C}$ with temperature interval of $50^{\circ} \mathrm{C}$. The experiments were repeated on three samples to check the reproducibility of the reported results.

\section{Results}

In Fig. 1 we show the PL of PS at nine different temperatures. The intensity scale in the figure is same for all spectra and hence one can clearly see the nonmonotonic variation of intensity of PL with temperatures. The measured PL spectra can be very well fitted by Gaussian profile as can be seen in the lowest spectrum of Fig. 1, corresponding to PL of as-prepared PS $\left(\mathrm{T},=25^{\circ} \mathrm{C}\right)$. The peak positions and intensities (area under the band) of PL as obtained from the non-linear least square fitting of the data with the Gaussian profile are shown in Fig. 2 as a function of annealing temperature T, The intensities are normalized with respect to that of the as-prepared PS. The solid lines in Fig. 2 have been drawn through the points as guide to the eye. It can be seen from Fig. 2(a) that the PL peak energy is $1.73 \mathrm{eV}$ in as-prepared PS and increases to $1.77 \mathrm{eV}$ at $\mathrm{T}_{a}=100^{\circ} \mathrm{C}$ and then gradually red-shifts to $1.68 \mathrm{eV}$ at $\mathrm{T},=600^{\circ} \mathrm{C}$. Above $600^{\circ} \mathrm{C}$ the peak energy increases and recovers to $1.74 \mathrm{eV}\left(\mathrm{T},=750^{\circ} \mathrm{C}\right)$. In Fig. 2(b) we see

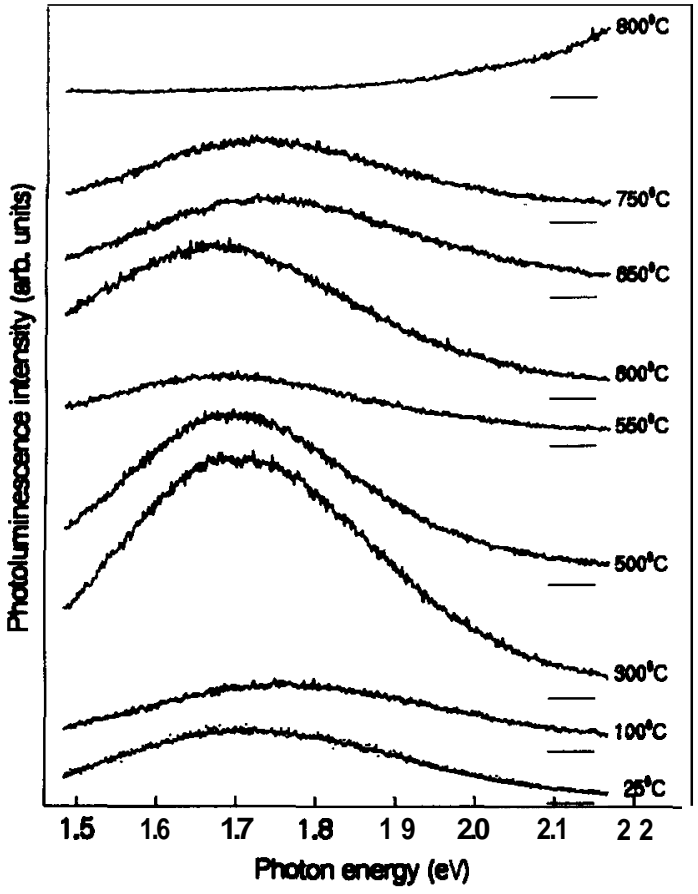

Fig. 1. Photoluminescence spectra of porous silicon at nine different annealing temperatures. Intensity scale of all spectra is same. Horizontal line on the right of each spectrum shows the zero of the intensity scale. In the lowest curve the dots represent experimental data and the solid line is the fitted Gaussian lineshape.

that the PL intensity remains constant till $\mathrm{T}$, reaches $100^{\circ} \mathrm{C}$ and then it increases upto $\mathrm{T}_{a}^{\prime}=350^{\circ} \mathrm{C}$, followed by a decrease. Above $550^{\circ} \mathrm{C}$ the intensity of $\mathrm{PL}$ from PS again starts increasing and then decreases for $\mathrm{T},>$ $700^{\circ} \mathrm{C}$. At $800^{\circ} \mathrm{C}$, the intensity vanishes completely.

The corresponding changes in Raman spectra as a function of annealing temperature are shown in Fig. 3. In the same figure we have also shown (top curve) the Raman spectrum of bulk crystalline $\mathrm{Si}$ (c-Si). Comparing the Raman spectra from PS at different $\mathrm{T}$, with that of $\mathrm{c}-\mathrm{Si}$, one can see that Raman line of the PS is red-shifted and highly asymmetric till $\mathrm{T},=550^{\circ} \mathrm{C}$. It is known that the asymmetric Raman line shape can arise due to the phonon confinement in the nanoscale crystallites [18]. In PS these can be either approximated to cylindrical (ie. quantum wire) or spherical in shape. In this model the Raman line shape is given by

$$
I_{c}(\omega)=A \int_{0}^{q_{\max }} \frac{d \vec{q}|c(0, \vec{q})|^{2}}{[\omega-\omega(\vec{q})]^{2}+\left(\Gamma_{c} / 2\right)^{2}}
$$

where $\omega(\vec{q})$ is the phonon dispersion curve for bulk silicon, which has been taken as $\omega(q)=\omega_{\circ}\left(1-0.18 q^{2}\right)$, which fits the experimental curve well in the direction of $\Gamma-X$ upto $q=0.8$. $\omega_{0}$ and $\Gamma_{c}$ are phonon frequency $(\vec{q}=0)$ and the natural linewidth of the bulk 


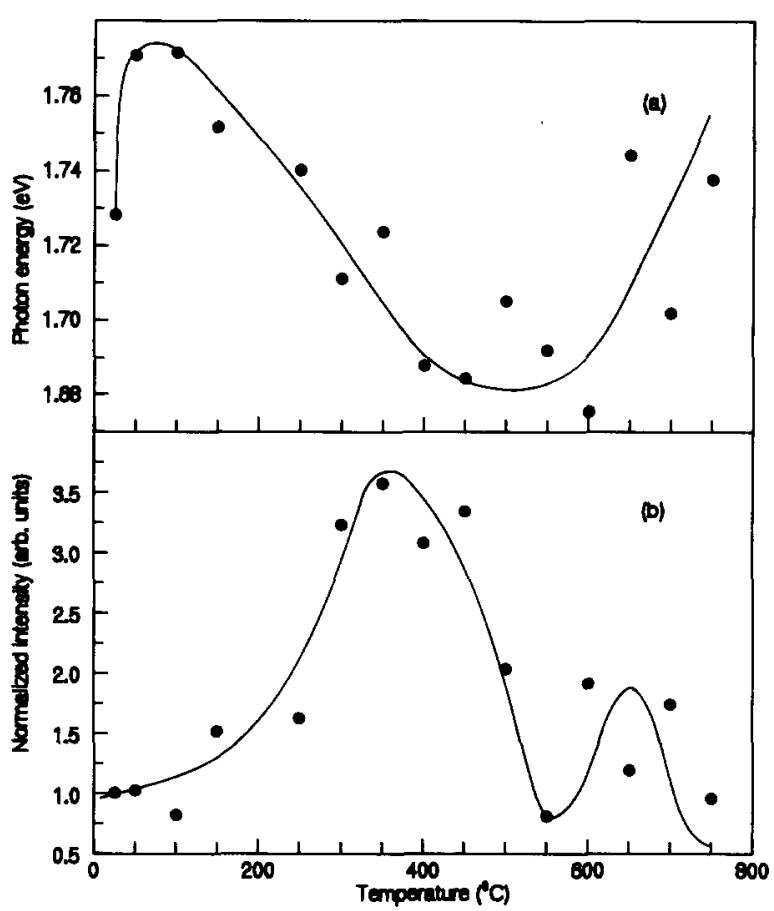

Fig. 2. The variation of the phtoluminescence (a) peak position and (b) intensity of porous silicon as a function of annealing temperature. The intensities are normalized with respect to that of as-prepared porous silicon. The solid lines through the data points are guide to the eye.

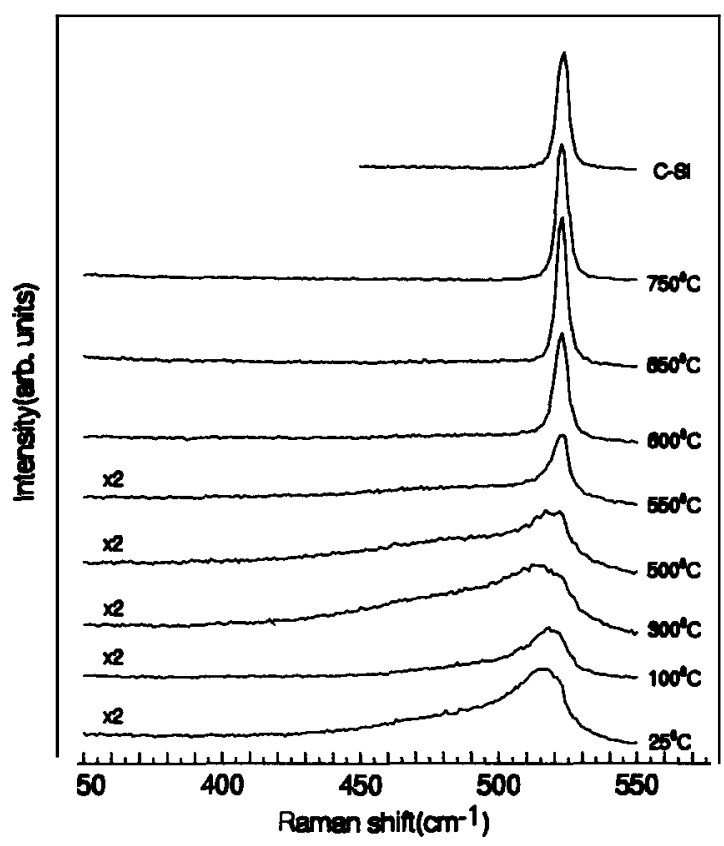

Fig. 3. Raman spectra of porous silicon at eight different annealing temperatures. The Raman line of the bulk crystalline $\mathrm{Si}$ is shown at the top.

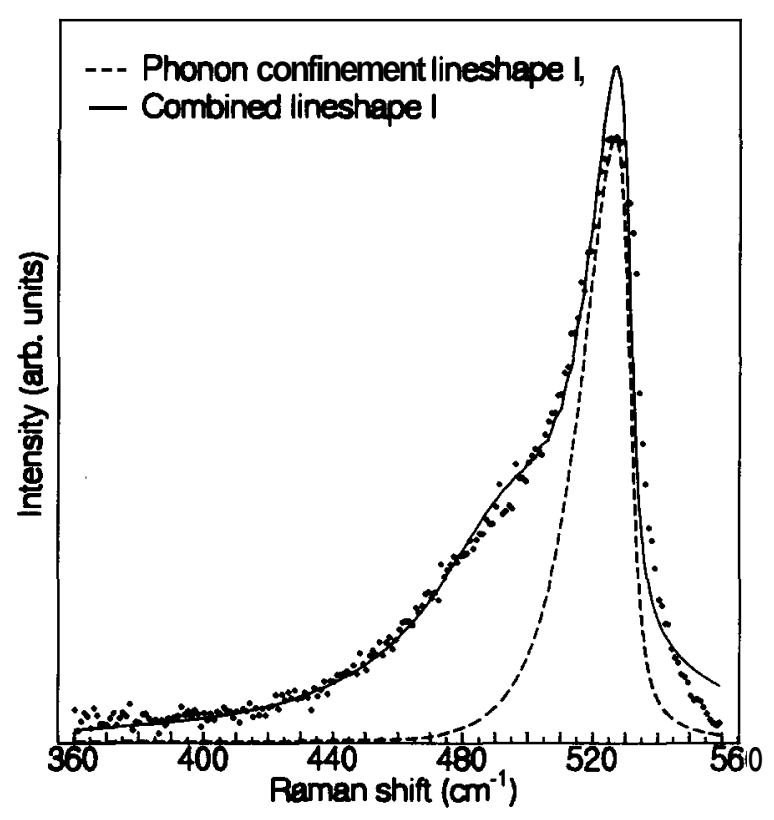

Fig. 4. Dots show the Raman spectrum of as-prepared porous silicon. The dashed line shows the fit using the confinement model alone $I_{c}(\omega)$. The solid line shows the best fit using $I(\omega)=I_{c}(\omega)+I_{d}(\omega)$ (a sum of phonon confinement effect and disorderd component).
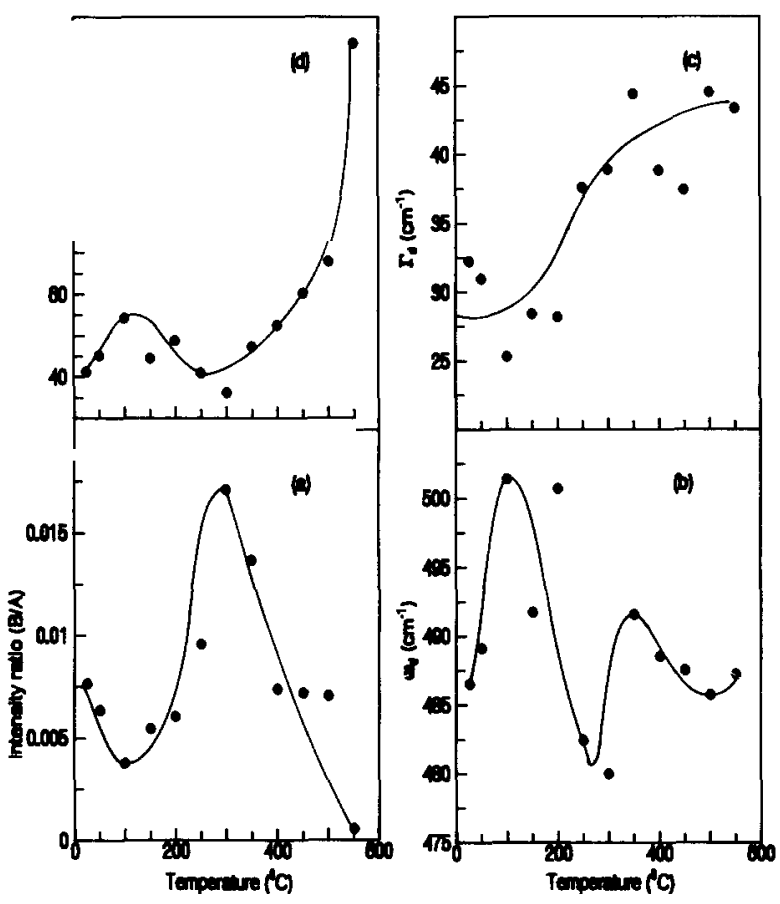

Fig. 5. Variation of the (a) the relative intensity of two components of Raman line, $B / A$ (b) Raman peak frequency of the disordered component, $\omega_{d}$ (c) Raman linewidth (HWHM) of the disordered component , $\Gamma_{d}$ (d) nanostructure diameter, $L$ with different annealing temperatures. The solid lines through the points are guide to the eye. 
silicon, the values of which have been taken as $\omega_{0}=$ $521.9 \mathrm{~cm}^{-1}$ and $\Gamma_{c}=4.7 \mathrm{~cm}^{-1}$. The wavevector $q$ has been expressed in units of $2 \pi / a$ (where a is the lattice constant of $\mathrm{Si}=5.4 \AA$ ) and $q_{\max }=0.8 . A$ is a constant. $c(0, \vec{q})$ is the Fourier coefficient of the phonon confinement function. One may note that the Raman line in nanoparticles is red-shifted and asymmetric towards the low frequency side because the phonon dispersion $\omega(\vec{q})$ in bulk silicon is a decreasing function of $\boldsymbol{q}$. We have used the phonon confinement function to be Gaussian which is found to be suitable for micro-crystalline Si : $|c(0, \vec{q})|^{2}=\exp \left[-q^{2} L^{2} /\left(4 a^{2}\right)\right], L$ is the dimension of the nanocrsytallite of Si. $L$ is the diameter of the cylinder for infinitely long cylindrical particles and diameter for spherical particles. It has been shown that for the PS, the phonon confinement in spherical particles explains the line shape better than with cylindrical particles $[19,12]$. Hence we have tried to fit the experimental Raman spectra from the PS using the above model with spherical particles. The best fit with diameter $L=42.7 \AA$ is shown by dashed line in Fig. 4 for the as-prepared PS and the corresponding value of $\chi^{2}$ is 2304. It is obvious from Fig. 4 that the phonon confinement model alone can not explain the PS Raman line shape.

In the next step, we attempted to fit the observed line shape with $I(\omega)=I_{c}(\omega)+I_{d}(\omega)$, where $I_{d}(\omega)=$ $B \Gamma_{d}\left[\left(\omega-\omega_{d}\right)^{2}+\Gamma_{d}^{2}\right]^{-1}$ is the Lorenzian line shape and arises due to disordered/amorphous component of the PS. Here $\omega_{d}$ and $\Gamma_{d}$ are the phonon frequency and half width at half maximum (HWHM), respectivly for the disordered component and $B$ is a constant. The presence of the disordered/amorphous phase has been reported earlier using EPR measurements [11]. Raman scattering measurements of microcrystalline silicon prepared in hydrogen plasma have shown crystalline as well as amorphous features. The latter has been attributed to a surface-like $\mathrm{Si}$ - $\mathrm{Si}$ shearing mode at the grain boundaries of the microcrystallites [20]. In the above exercise of nonlinear least square fit of the data with $I(\omega)$, the fitting parameters are $L, \omega_{d}, \Gamma_{d} \boldsymbol{A}$ and $\boldsymbol{B}$. In Fig. 4 we have plotted the fitted $I(\omega)$ (solid line) and the corresponding value of $\chi^{2}$ is 350 . The value of $L$ is the same $(42.7 \AA)$ as obtained by the previous fit with $I_{c}(\omega)$ alone. It can be seen from Fig. 4 and the lower value of $\chi^{2}$ that the Raman line shape $I(\omega)=I_{c}(\omega)+I_{d}(\omega)$ fits extremely well the experimental spectrum. In Fig. 5(a) we show the relative intensity $B / A$ of the two components $I_{d}(\omega)$ and $I_{c}(\omega)$. In Fig. $5(\mathrm{~b}-\mathrm{d})$ we have shown the variation of $\omega_{d}, \Gamma_{d}$ and $L$ with different $T_{a}$. The solid lines through the points are guide to the eye.

\section{Discussion}

Our experimental results can not be explained in terms of either of the two models mentioned earlier. Instead a new hybrid model [14] may be more suitable to understand the experimental data. According to this model the incident radiation creates electron-hole pairs in nanoscale structures present in the PS but these carriers, unlike in quantum confinement model, do not radiatively recombine inside the nanoparticles. Instead, these carriers diffuse out and recombine through various luminescent centres. These centres can be point defects located at the $\mathrm{Si} / \mathrm{SiO}_{2}$ interface or in the thin $\mathrm{SiO}_{2}$ layers or complexes of silicon with $\mathrm{O}, \mathrm{H}$ or $\mathrm{F}$ formed on the surface of the nanoparticles. The dominance of any of these luminescence centres will depend on the anode-etching conditions, post-preparation and thermal treatments. This can rationalize the different reports on variations of the PL characteristics in the PS. In our experiments, initial increase in the PL intensity with annealing temperature in Fig. 2(b) suggests an increase in defect density during thermal annealing. As PS is heated beyond $350^{\circ} \mathrm{C}$, hydrogen desorbs from the sample as seen in EPR studies (via increase in dangling bonds) and infra-red absorption measurements [11]. This will decrease the defects related to $\mathrm{Si}-\mathrm{H}$ complexes at the surface of the nanostructures and hence the PL intensity. This can also cause a decrease in the PL peak position. Further heating $\left(\mathrm{T},>500^{\circ} \mathrm{C}\right)$ results in the repassivation of the dangling bonds due to formation of $\mathrm{SiO}_{2}$. The luminescence now can be due to the defects at $\mathrm{Si} / \mathrm{SiO}_{2}$ interface or the defects in $\mathrm{SiO}_{2}$ and can result in higher PL peak position as seen in Fig. 2(a). Now, coming to Raman scattering results, we note the following : Variation of the relative intensity $(B / A)$ [ intensity of $I_{d}(\omega)$ /intensity of $\left.I_{c}(\mathrm{w})\right]$ in Fig. 5(a) agrees with the above conclusion that the disorder in the form of possible $\mathrm{Si}-\mathrm{H}$ complexes first increases up to $350^{\circ} \mathrm{C}$ and then decreases. The variation in $\omega_{d}$ and $\Gamma_{d}$ in Fig. $5(\mathrm{~b})$ and (c) with annealing temperature suggests that composition and structure of the $\mathrm{Si}-\mathrm{H}$ complexes are temperature sensitive coroborating the corresponding changes in the PL peak position. The drastic change in the Raman line shape for $\mathrm{T}_{a}>550^{\circ} \mathrm{C}$ can arise because increasing portions of nanoscale $\mathrm{Si}$ get converted into $\mathrm{SiO}_{2}$. The fact that the $\mathrm{SiO}_{2}$ has much less absorption coeffecient of the incident radition at $2.41 \mathrm{eV}$ (as observed in the shift of the absorption band at $\mathrm{T}_{\mathrm{a}} \sim 600^{\circ} \mathrm{C}$ [21]) the Raman signal will be dominated by the underlying substrate. Fig. 5(d) suggests that the size of the nanoscale unit changes with annealing temperature. The initial increase up to $100^{\circ} \mathrm{C}$ seems to be correlated with the increase of $\omega_{d}$ [Fig. 5(b)] from 487 to $502 \mathrm{~cm}^{-1}$, similar to the reported dependence of the peak of the amorphouslike Raman component on the size of the microcrystallite of $\mathrm{Si}[20]$. More quantitative TEM studies are required to look into this aspect.

In conclusion, the characteristics of both the nanostructures and the defect states responsible for PL in the PS have been investigated as a function of thermal annealing. PL experiments help in understanding the nature of the luminescent states whereas Raman scattering probes the underlying quantum network. A hybrid model incorporating the features of nanoscale units acting as a reservoir of the electron-hole pairs and silicon complexes (with $\mathrm{O}, \mathrm{H}$ and $\mathrm{F}$ ) and defects located outside the nanoparticles as radiative luminescent centres can explain our experimental results reasonably well.

Acknowledgement - One of us (AKS) thanks Department of Science and Technology for financial support. 


\section{References}

[1] M. S. Brandt, €1. D. Fuchs, M. Stutzmann, J. Weber and M. Cardona, Solid State Commun. 81, 307 (1992)

[2] L. E. Friedersdorf, P. C. Searson, S. M. Prokes, O. J. Glembocki and J. M. Macaulay, Appl. Phys. Lett. 60,2285 (1992).

[3] M. B. Robinson, A. C. Dillon, D. R. Haynes and S. M. George, Appl. Phys. Lett. 61, 1414 (1992).

[4] D. J. Wolford, B. A. Scott, J. A. Reimeer and J. A. Bradley, Physica 117B and 118B, 9220 (1983).

[5] L. T. Canham, Appl. Phys. Lett. 57, 1046 (1990).

[6] A. J. Read, R. J. Needs, K. J. Nash, L. T. Canham, P. D. J. Calcott and A. Qteish, Phys. Rev. Letts. 69, 1232 (1992).

[7] S. V. Bhat, K. Jayaram, D. V. S. Muthu and A. K. Sood, Appl. Phys. Lett, 60,2116 (1992).

[8] X. L. Zheng, W. Wang and H. C. Chen, Appl. Phys. Lett. 60,986 (1992).

[9] Z. Y. Xu, M. Gal and M. Gross, Appl. Phys. Lett. 60, 1375 (1992).

[10] K. H. Li, C. Tsai, J. C. Campbell, B. K. Hance and J. M. White, Appl. Phys. Lett. 62,3501 (1993).

[11] S. M. Prokes, W. E. Carlos and V. M. Bermudez, Appl. Phys. Lett. 61, 1447 (1992).
[12] A. K. Sood, K. Jayaram and D. Victor S. Muthu, J. Appl. Phys. 72,4963 (1992).

[13] W. Zhou, H. Shen, J. F. Harvey, R. A. Lux, M. Dutta, F. lu, C. H. Perry, R. Tsu, N. M. Kalkhoran and F. Namavar, Appl. Phys. Lett. 61, 1435 (1992).

[14] G. G. Qin and Y. Q. Jia, Solid State Commun., 86, 559 (1993)

[15] S. M. Prokes, O. J. Glembocki, V. M. Bermudez, R. Kaplan, L. E. Friedersdorf and P. C. Searson, Phys. Rev. B 4513788 (1992).

[16] A. Roy, A. Chainani, D.D.Sarma and A.K. Sood, Appl. Phys. Lett. 61, 1665 (1992).

[17] V. K. Jain, Amita Gupta, A. Kumar, G. K. Singhal, O.P. Arora, T.Srinivasan, D. S. Ahuja, P. Puri, and Vikram Kumar, Bull. Mater, Sci. 16, 239 (1993).

(18] I. H. Campbell and P. M. Fauchet, Solid State Commun. 58, 739 (1986).

(19] Z. Sui, P. P. Leong, I. P. Herman, G. S. Higashi and H. Temkin, Appl. Phys. Lett. 60, 2086 (1992).

[20] Z. Iqbal and S. Vepfek, J. Phys. C = Solid State Phys. 15, 377 (1982).

[21] V. Petrova-Koch, T. Muschik, A. Kux, B. K. Meyer, F. Koch and V. Lehmenn, Appl. Phys. Lett. 61,943 (1992). 